\title{
Cultural adaptation and psychometric evaluation of the Polish version of the Newcastle Satisfaction with Nursing Scale
}

\author{
Danuta Dyk ${ }^{1}$, Aleksandra Gutysz-Wojnicka², Edyta K. Cudak', Dorota Talarska ${ }^{3}$
}

\begin{abstract}
'Department of Anaesthesiological and Intensive Care Nursing, Poznan University of Medical Sciences, Poznan, Poland

2Department of Nursing, University of Warmia and Mazury, Olsztyn, Poland ${ }^{3}$ Laboratory of Social Nursing, Department of Health Promotion, Poznan University of Medical Sciences, Poznan, Poland
\end{abstract}

Submitted: 25 January 2012

Accepted: 22 April 2012

Arch Med Sci 2014; 10, 4: 782-790

DOI: 10.5114 /aoms.2014.44870

Copyright (c) 2014 Termedia \& Banach

\section{Abstract}

Introduction: The paper presents the methods of cultural adaptation of the Newcastle Satisfaction with Nursing Scale (NSNS) to the conditions in Polish hospitals.

Material and methods: The process of cultural adaptation of the research tool took into consideration an analysis of different equivalence levels, the translation procedure and the estimation of psychometric parameters. The Polish version of the NSNS questionnaire was correctly completed by 787 patients making up $59.36 \%$ of the total number of patients who received the scale.

Results: The Polish version of the NSNS questionnaire was correctly completed by 787 patients making up $59.36 \%$ of the total number of patients who received the scale. Cronbach's $\alpha$ coefficient was 0.921 for the "experience" scale and 0.981 for the "satisfaction" scale. The values of Spearman's rank correlation coefficient were from 0.224 to 0.797 for "experience" and 0.815-0.894 for "satisfaction". All questionnaire items of the Polish NSNS version exerted a statistically significant influence on the total results of the scale $(p=0.0001)$.

Conclusions: The Polish NSNS version, similarly as the original version, can identify differences referring to "experience" and "satisfaction" with nursing care between the particular departments and between hospitals. The Polish NSNS version was conducted among patients during multicentre studies and it meets the criteria of functional, psychometric and façade equivalences.

Key words: cultural adaptation, psychometric evaluation, the Newcastle Satisfaction with Nursing Scale.

\section{Introduction}

Each research tool being the result of human scientific activity is developed in the cultural context of its author, and it is designed for research purposes for the people belonging to a given culture. A tool applied without an intercultural comparison will probably contain many features characteristic for the culture of its origin; therefore, it cannot be applied directly in its original form to any other cultural environment [1].

\author{
Corresponding author: \\ Edyta K. Cudak \\ Department \\ of Anaesthesiological \\ and Intensive Care Nursing \\ Poznan University \\ of Medical Sciences \\ 11 Smoluchowskiego St \\ 60-179 Poznan, Poland \\ Phone: +48 616559239 \\ Fax: +48 616559238 \\ E-mail: edytacud@ump.edu.pl
}


Therefore, cultural adaptation of a research tool has to encompass all activities connected with its adaptation to the cultural context of people to whom it is to be applied. In order to achieve this goal, before the beginning of the adaptation procedure of any given tool, it is recommended to carry out an analysis of different equivalence levels starting with the most general ones [1]:

- Equivalence of indicators. The studied independent variable is estimated using indicators or features reflecting the accepted conception of a given notion. This variable evaluated in the tool should be assessed on the basis of the particular tool's items (questions), whether they express an identical idea and situation in both cultures.

- Equivalence of results consists in such adaptation of the tool that people from both cultures (the original and the target one) obtain the same result and the same level of the investigated variable.

- Equivalence referring to the investigation conditions. In some situations, the testing tool's results depend on factors such as familiarity with tests and testing procedures in general, motivation to cooperate with a given testing procedure and a researcher or questionnaire distributor and also on the researcher's personality.

- Equivalence of test in terms of factor analysis.

- Equivalence in terms of the psychometric properties of the tool and the reliability of the adapted version of the tool.

Cultural adaptation of the diagnostic tool is rational only in cases when both cultures (the original and the target one) are comparable [1].

In intercultural studies, translation of the research tool is crucial. Much attention is devoted to semantic equivalence of both versions of the scale. In the translation of particular words and terms, apart from basic semantic meaning, there are other features of great importance such as possible meaning resulting from emotional association of a given word, its social context, or the approach of the tool's author to a given subject represented by a given word. Even the grammatical structure of the sentence, the use of verb tenses, or word order in the sentence can be of great significance [2-4].

Principles of Good Practice for the Translation and Cultural Adaptation Process for PatientReported Outcomes (PRO) Measures contains 10 steps to follow [5]:

- Step 1. Preparation - the period preceding the translation process includes obtaining the consent to use the research tool, an explanation of how to use the research tool.

- Step 2. Forward translation - translation of the original version into the target language; several versions are proposed by independent translators.

- Step 3. Reconciliation - a comparison of translated versions and selection of final version.

- Step 4. Backward translation - a re-translation from the new version into the language of the original by an independent translator.

- Step 5. Backward translation review - a comparison of backward translation to the original to ensure the same quality as the original version of the research tool.

- Step 6. Harmonization/correspondence-a comparison of backward translation to the original to identify discrepancies between the two language versions during translation ensuring equivalence of the target version.

- Step 7. Cognitive debriefing - evaluation/testing of the tool on a small group (pilot study) to ensure comprehension of the tool and cultural relevance of the translation.

- Step 8. Review of Cognitive Debriefing Results and Finalization - a comparison of the comprehension and interpretation of the tool with the original version.

- Step 9. Proofreading - a final review of the tool and correction of grammatical and stylistic mistakes.

- Step 10. Final Report - the report includes a description of methodology used and all translation decision undertaken throughout the process [5].

In order to ensure fine quality of the translation, methodologists recommend one to have the translated version verified by other translators who did not participate in the translation of the tool and by linguistic experts who can reliably verify grammatical and stylistic correctness of the adapted version [4].

For the development of nursing care as a scientific branch, it is important to carry out investigations using reliable and verified instruments [6-8]. Among many available instruments for the measurement of patient satisfaction with nursing care, only a few take into consideration the patient's approach to this issue, and the instrument's psychometric properties have not always been evaluated [9-11].

The Newcastle Satisfaction with Nursing Scale (NSNS) is a tool that meets the above-mentioned criteria. It is a statistically verified instrument constructed on the basis of previously verified aspects of nursing care that are important for patients [12]. The authors separated the aspects referring to nursing care from the total care offered in the hospital and they defined the criteria how to measure them. The prepared tool turned out to be adequately reliable and sensitive in detecting differences in the level of nursing care seen from the patient's point of view 
rather than from the personnel's perspective. The NSNS is designed principally to detect differences between wards and patient groups with respect to the total experience and satisfaction with nursing. Therefore, the analysis is primarily focused on the total scale scores [12]. The NSNS instrument has been used in the surgical and non-surgical hospital departments [12, 13]. Validation of this instrument and the measurement of the satisfaction with nursing care for female patients at an obstetric ward were carried out in 2005 in Canada [14]. The NSNS adaptation was done in Turkey, where the reliability and validity of the modified Turkish version of the NSNS were assessed [15].

The aim of the study was to adapt the Newcastle Satisfaction with Nursing Scale to Polish conditions as well as to evaluate psychometric properties of its Polish version.

\section{Material and methods}

\section{Development of the Polish version of the Newcastle Satisfaction with Nursing Scale}

After obtaining the consent from the authors of the original version, we focused on the problem of cultural adaptation of the research tool to Polish conditions [3] and on the necessity to respect the equivalence criteria [16]. In respect to functional and conceptual analyses, we have analyzed the following:

- Is "satisfaction with nursing care" coherent/ corresponding in both cultural areas (English and Polish)?

- Do aspects of nursing care that are important for patients, identified in the original scale, represent "good" and "poor" nursing care in both linguistic areas?

In order to find answers to these questions, a survey of Polish literature was carried out and the databases of the Main Medical Library in Warsaw and Medline were also used.

The search key words included: patient's satisfaction, patient's satisfaction with nursing care, measurement of satisfaction with nursing care, expectations of patients in relation to nurses in Poland, tools for the measurement of satisfaction with nursing care [17-19]. No limitations were applied to the publication dates of particular papers.

In order to preserve as exact as possible semantic equivalence in the translation of the NSNS instrument into Polish, the translation was done by two different and independent translators. Then, experts developed one common Polish version of the NSNS. The Polish version of the NSNS was translated back (backward translation) into English by another translator without any reference to the English original. The retranslated English version was sent to the English co-author of the original tool for revision. Consequently, some minor corrections had to be introduced, and the final Polish version of the Newcastle Satisfaction with Nursing Scale (the Polish version of the NSNS) was approved.

The Polish version of the NSNS is composed of three parts:

Part I of the scale - experiences related to nursing care - includes 26 statements (15 positive and 11 negative) with the answer option based on a 7-degree Likert scale. The analysis of the answers helps to determine the general score of "experience" within the 0-100 range with 100 points reflecting the best possible experience of the nursing care and 0 points reflecting the worst possible experience.

Part II of the scale - satisfaction with the nursing care - includes 19 statements with the answer option on a 5-degree Likert scale ranging from "completely satisfied" to "completely unsatisfied". The analysis of the answers helps to determine the general score of "satisfaction" within the 0-100 range with 100 points reflecting full satisfaction with all aspects of nursing care and 0 points reflecting lack of satisfaction with any aspect of nursing care.

Part III of the scale - includes questions on patients' demographics and hospital stay and two questions on the general satisfaction with nursing care during the whole period of hospitalization:

- question 6 - How would you rate the nursing care you received in this ward?

- question 7 - Overall how would you rate your recent stay in this ward?

Both questions could be answered on a 9-degree Likert scale.

During the practical application of the corrected questionnaire, the patients were encouraged to express their opinions and remarks referring to the particular items and methods of responding. These opinions were entered by the respondents in Part 3 of the questionnaire.

Also, the research procedure was identical to the instructions given by the English authors and the graphical form of the questionnaire was identical with the original tool [12].

\section{Application of the Polish version of the Newcastle Satisfaction with Nursing Scale to Polish surgical and non-surgical patients}

The study was conducted in six hospitals in Poland, including three district hospitals, two provincial hospitals and one university hospital. The data were collected in the years 2006-2008. We aimed to gather data from 100 patients in each ward. The sample size calculations were based on previous results of the study on the development of 
the original version of the Newcastle Satisfaction with Nursing Scale [12]. We also aimed to conduct the study at least in one surgical department and in one non-surgical department in each hospital. The research procedure was prepared according to the instructions provided by the authors of the original version of the instrument [12].

Following the decision of the authors, absence of up to $5 \%$ of responses was acceptable [12].

\section{Statistical analysis}

Because experience and satisfaction scale scores were not equally distributed, nonparametric tests were applied. Statistical methods of data analysis were applied separately for results on the "experience" and "satisfaction" scales of the Polish NSNS version. The following methods were applied: descriptive statistics, methods of dependence analysis (Spearman's rank correlation coefficient), methods of statistical conclusion drawing (Mann-Whitney test, Kruskal-Wallis ANOVA test) [20].

In order to estimate the ability of the Polish NSNS version to detect differences in the levels of "experience" and "satisfaction" between hospitals and departments, Dunn's test of multiple comparisons was applied.

Criteria were established as follows: Cronbach's reliability $\alpha$ coefficient - values of 0.7 and more were accepted as values confirming reliability of the scale; Spearman's rank correlation coefficient - values of 0.4 and more were accepted as confirmation of the influence of a given scale item on the general result.

Reliability of the scale was analyzed on the basis of the questionnaires with all items answered. The next step in the verification was an attempt to improve the scale's reliability on the basis of Cronbach's $\alpha$ coefficient obtained when a given item was removed.

Content validity of the Polish NSNS version was studied by analyzing the responses to particular items of the questionnaire. It was assumed that when no answers to particular items were obtained from more than $5 \%$ of respondents, the item did not meet the accuracy criteria [12].

The convergence aspect of validity was analyzed by investigating the correlation of the "experience" result and the "satisfaction" result with the results of external criteria. Replies to two questions in part III of the scale were used as an external criteria measurement.

In order to estimate this dependence, Spearman's rank correlation coefficient was applied.

Construct validity was determined by comparing the results obtained in the "experience" and the "satisfaction" scales with the results of "the known groups validity". Based on a literature review, it was found that elderly patients who had known their primary nurse evaluated their nursing care more highly $[9,12,15,21]$. This study investigates the effect of age, sex and education on the levels of "experience" and "satisfaction" in order to confirm the results obtained by other authors. Finding a similar relation in our study confirmed the theoretical accuracy of the tool. It was investigated how age, gender, education and the fact of having a primary nurse influenced the patients' level of "experience" and "satisfaction". In the tests used, the significance level was accepted as $\alpha=0.05$.

\section{Results}

Data were obtained from nine departments in six hospitals. The total number of completed questionnaires was 918; however, only 787 (85\%) questionnaires were filled in correctly.

Men constituted $51 \%$ of the respondents, with the average age of 52 years. The major group of the respondents (46\%) had vocational education while $25 \%$ had secondary education.

In the Polish literature, the most important types of patient's needs - in the context of nursing care quality - are mentioned. They include the need for information, control of the situation, safety, interest in the patient's problems and emotional support, respect and acceptance, intimacy and confidence, and friendly atmosphere [22]. Other aspects of nursing care evaluated during studies on patient's satisfaction with nursing care include: correct performance of interventions, interpersonal relations, subjective treatment, readiness to inform the patient, education, satisfying existential needs, and proper accommodation conditions [2]. Polish patients defined their expectations regarding nursing care, but they did not determine what they understood by "good quality" nursing care. However, it must be admitted that the majority of nursing care aspects identified by patients in England have been confirmed in Poland as well.

\section{Evaluation of the Polish NSNS version by patients}

Analysis of the responding method to particular questionnaire parts facilitated identification of the most frequently unanswered items. Lack of response might be because of unclear, too difficult wording, not adequate to the situation or evoking negative emotions. As for the "experience" scale, item no. 18 was most frequently left unanswered ("Nurses informed the next shift nurse what was happening with my care"). In total, the blank response rate to this question was $2.40 \%$.

In the "satisfaction" scale, the patients most frequently skipped item no. 11 ("How nurses helped to put your relatives' or friends' minds 
at rest"). To that item, there was no response in $6.50 \%$ of questionnaires.

The ability of the Polish NSNS version to detect differences in patients' experiences and satisfaction with nursing care

The Polish version of the scale detects the differences in experiences and satisfaction with nursing care between wards and hospitals. Statistically significant variations in care levels were also evident when the results of different wards within the same hospital were compared.

A comparison of the significance result in the "experience" scale was obtained in particular hospitals and departments by using the Kruskal-Wallis test $\mathrm{H}(8, n=895)=392.3764$ - between hospitals $p=0.0001$, between departments $p=$ 0.0001 . A comparison of the results obtained in particular departments indicated that in the majority of compared departments, significant statistical differences were present. Regarding the "satisfaction" scale, the comparison of the results obtained between particular departments showed that the statistical value of the Kruskal-Wallis test was $\mathrm{H}(8, n=883)=366.9461, p=0.0001$. A comparison of results in particular hospitals, the Krus kal-Wallis test showed $\mathrm{H}(5, n=883)=140.8314$, $p=0.0001$ between hospitals.

\section{Evaluation of the psychometric properties of the Polish NSNS version}

The reliability of the "experience" scale for $n=$ 787. Cronbach's a coefficient was 0.921. Increase of Cronbach's coefficient was possible when item no. 6 ("Nurses gave me information just when I needed it") was eliminated, which resulted in an increase of the coefficient to the value of 0.928 . In turn, elimination of item no. 22 ("Nurses had time to sit and talk to me") caused an increase of Cronbach's $\alpha$ coefficient to the value of 0.922 . In cases when any other item was removed, the coefficient value was maintained at an unchanged level, or decreased, with the lowest value at 0.916. Cronbach's $\alpha$ coefficient of the "satisfaction" scale was 0.981 . The removal of any test items did not cause any increase of the coefficient value. Having analyzed all correctly completely questionnaires ( $n=$ 787), we found that all items had an effect on the value of the "experience" scale, although the coefficient values were below 0.40 for items 6 and 22 (Table I).

In the investigated institutions, the values of Spearman's rank correlation coefficient for particular items of the "satisfaction with nursing" scale reached above the 0.40 value. All items exerted a significant effect on the values of the "satisfaction" scale (Table II).
Analysis of the connection between the results of the Polish NSNS version and external criteria (convergence aspect of validity)

In the studies on the validity of the NSNS, the responses to item 6 ("How would you rate the nursing care you received in this ward?") and item 7 ("Overall how would you rate your recent stay in this ward?") from Part 3 of the NSNS questionnaire were used as external criteria.

In a clear majority of the studied institutions, a significant dependence was found between the results obtained in the "experience" scale and in the response to item 6 from Part 3. The value of Spearman's rank correlation coefficient ranged from 0.253 to 0.788 ; in general (for all departments) it was $r=0.632$ and $p=0.0001$.

Analyzing the correlation between the results of the "experience" scale and the results of item 7 in Part 3 ("Overall how would you rate your recent stay in this ward?"), a statistically significant correlation was found for all departments ( $r=$ 0.612 and $p=0.0001)$. In particular departments the value of Spearman's rank correlation coefficient ranged from 0.227 to 0.725 . In the estimation of the dependence between the results of the "satisfaction" scale and the results of external criteria (Part 3. item 6 "How would you rate the nursing care you received in this ward?"), the value of Spearman's rank correlation showed values from 0.373 to 0.836 ; in general $r=0.738$ and $p=$ 0.0001 . A correlation was found between the results of the "satisfaction" scale and the answers to question no. 7 in part 3. When estimating the results from the "satisfaction" scale and the results of external criteria (Part 3, item 7 "Overall how would you rate your recent stay in this ward"), a statistically significant dependence $(p=0.0001)$ was found in all studied hospitals. The value of the correlation coefficient ranged between 0.372 and 0.797; in general $r=0.701$ and $p=0.0001$.

\section{Analysis of known group validity}

In Poland, the study results confirmed a significant influence of the patient's age on the results of the "experience" scale $(p=0.0005)$ and "satisfaction" scale $(p=0.0194)$. Patients over the age of 60 gave the highest scores pertaining to their experiences, while patients under the age of 40 gave lower scores. Patients under the age of 40 assessed satisfaction with nursing care significantly lower than patients in the 40 to 60 group.

Also gender influenced the patient's results of "experience" ( $p=0.000042)$ and "satisfaction" $(p=0.0001)$. Men reached higher values for both the "experience" and "satisfaction" scale. There 
Table I. Item-total correlation for "experience with nursing care scale"

\begin{tabular}{|c|c|c|}
\hline Questions & $\begin{array}{c}\text { Spearman's rank } \\
\text { correlation coefficient }\end{array}$ & Value of $p$ \\
\hline 1. It was easy to have a laugh with the nurses. & $0.549^{* *}$ & 0.0001 \\
\hline 2. Nurses favored some patients over others. & $0.711^{\star *}$ & 0.0001 \\
\hline 3. Nurses did not tell me enough about my treatment. & $0.598^{\star \star}$ & 0.0001 \\
\hline 4. Nurses were too easy going and laid back. & $0.712^{* *}$ & 0.0001 \\
\hline 5. Nurses took a long time to come when they were called. & $0.696^{* *}$ & 0.0001 \\
\hline 6. Nurses gave me information just when I needed it. & $0.224^{\star \star}$ & 0.0001 \\
\hline 7. Nurses did not seem to know what I was going through. & $0.671^{\star *}$ & 0.0001 \\
\hline 8. Nurses turned the lights off too late at night. & $0.671^{\star *}$ & 0.0001 \\
\hline 9. Nurses made me do things before I was ready. & $0.704^{\star *}$ & 0.0001 \\
\hline 10. No matter how busy nurses were, they made time for me. & $0.626^{* *}$ & 0.0001 \\
\hline 11. I saw nurses as friends. & $0.681^{* *}$ & 0.0001 \\
\hline 12. Nurses spent time comforting patients who were upset. & $0.714^{\star *}$ & 0.0001 \\
\hline 13. Nurses checked regularly to make sure I was okay. & $0.686^{* *}$ & 0.0001 \\
\hline 14. Nurses let things get on top of them. & $0.708^{* *}$ & 0.0001 \\
\hline 15. Nurses took no interest in me as a person. & $0.745^{* *}$ & 0.0001 \\
\hline 16. Nurses explained what was wrong with me. & $0.459^{* *}$ & 0.0001 \\
\hline 17. Nurses explained what they were going to do to me before they did it. & $0.711^{\star *}$ & 0.0001 \\
\hline 18. Nurses told to the next shift what was happening with my care. & $0.660^{* *}$ & 0.0001 \\
\hline 19. Nurses knew what to do without relying on doctors. & $0.652^{\star *}$ & 0.0001 \\
\hline 20. Nurses used to go away and forget what patients asked for. & $0.713^{* *}$ & 0.0001 \\
\hline 21. Nurses made sure that patients had privacy when they needed it. & $0.727^{\star *}$ & 0.0001 \\
\hline 22. Nurses had time to sit and talk to me. & $0.388^{* *}$ & 0.0001 \\
\hline 23. Doctors and nurses worked well together as a team. & $0.711^{\star \star}$ & 0.0001 \\
\hline 24. Nurses did not seem to know what each other was doing. & $0.757^{\star *}$ & 0.0001 \\
\hline 25. Nurses knew what to do for the best. & $0.744^{* *}$ & 0.0001 \\
\hline 26. There was a happy atmosphere in the ward, thanks to nurses. & $0.717^{\star \star}$ & 0.0001 \\
\hline
\end{tabular}

was no impact of education on the result of "experience" ( $p=0.2204)$ or "satisfaction" $(p=1075)$.

\section{Discussion}

The objective of the studies was development and application of a research instrument for measuring patient satisfaction with nursing care in Poland. Such a tool must have adequate psychometric properties and it should be constructed on the basis of nursing care attributes essential for the patients. It should identify particular aspects of nursing care and be constructed in an easy and attractive form for a patient $[23,24]$.

Taking into consideration a wide range of sources dealing with the construction of a model instrument evaluating patient "satisfaction" with nursing care properly and reliably, we selected the NSNS as being potentially best suited for application in Poland. The advantage of the NSNS tool is that it is designed to measure the patient's "experience" and "satisfaction" with nursing care. The disadvantage of the NSNS tool is that, despite being based on prior identification of essential attributes of "good quality nursing care" by the patients, the factor analyses did not identify specific aspects of nursing care. Lack of this information did not allow the creation of any sub-scales evaluating dimensions of nursing care. The result of the NSNS must be interpreted as a general result of satisfaction with nursing care in a given department. Patient 
Table II. Item-total correlation for "satisfaction with nursing care scale"

\begin{tabular}{|c|c|c|}
\hline Questions & $\begin{array}{l}\text { Spearman's rank } \\
\text { correlation coefficient }\end{array}$ & Value of $p$ \\
\hline 1. The amount of time nurses spent with you. & $0.815^{* *}$ & 0.0001 \\
\hline 2. How capable nurses were at their job. & $0.829^{\star *}$ & 0.0001 \\
\hline 3. There was always a nurse around if you needed one. & $0.873^{\star \star}$ & 0.0001 \\
\hline 4. The amount nurses knew about your care. & $0.875^{* *}$ & 0.0001 \\
\hline 5. How quickly nurses came when you called for them. & $0.863^{* *}$ & 0.0001 \\
\hline 6. The way the nurses made you feel at home. & $0.874^{\star *}$ & 0.0001 \\
\hline $\begin{array}{l}\text { 7. The amount of information nurses gave to you about your } \\
\text { condition and treatment. }\end{array}$ & $0.837^{\star *}$ & 0.0001 \\
\hline 8. How often nurses checked to see if you were okay. & $0.884^{\star \star}$ & 0.0001 \\
\hline 9. Nurses' helpfulness. & $0.870^{* *}$ & 0.0001 \\
\hline 10. The way nurses explained things to you. & $0.868^{* *}$ & 0.0001 \\
\hline 11. How nurses helped to put your relatives' or friends' minds at rest. & $0.874^{* *}$ & 0.0001 \\
\hline 12. Nurses' manner in going about their work. & $0.871^{* *}$ & 0.0001 \\
\hline $\begin{array}{l}\text { 13. The type of information nurses gave to you about your } \\
\text { condition and treatment. }\end{array}$ & $0.857^{* *}$ & 0.0001 \\
\hline 14. Nurses' treatment of you as an individual. & $0.880^{\star *}$ & 0.0001 \\
\hline 15. How nurses listened to your worries and concerns. & $0.887^{* *}$ & 0.0001 \\
\hline 16. The amount of freedom you were given on the ward. & $0.818^{\star *}$ & 0.0001 \\
\hline 17. How willing nurses were to respond to your requests. & $0.894^{\star *}$ & 0.0001 \\
\hline 18. The amount of privacy nurses gave you. & $0.848^{\star \star}$ & 0.0001 \\
\hline 19. Nurses' awareness of your needs. & $0.885^{\star *}$ & 0.0001 \\
\hline
\end{tabular}

${ }^{* *} p<0.01$

satisfaction is a psychological phenomenon that cannot be observed and measured in a direct way. The measurement of patient satisfaction requires a precise definition of its meaning and determination of its indicators reflecting the accepted concept. The authors of the NSNS tool accepted, according to Pascoe [25], that patient satisfaction is a reaction to essential aspects of human experiences connected to received nursing care. The patient's experiences are subject to cognitive and rational judgment. Therefore, satisfaction is a cognitive process and emotional reaction. Such an understanding of the notion of "satisfaction" justifies the division of the NSNS tool into two parts: "experience" and "satisfaction" with nursing care. In Polish conditions, patient satisfaction is frequently understood as the difference between what the patient receives and what his or her individual expectations of nursing care are [22].

In other studies "patient satisfaction" is defined as some emotional reaction to an experience related to granted nursing care [11, 24, 26]. This proves an alignment in the interpretation of "patient satisfaction" between the cultural area where the NSNS originated and the area where it is to be applied.

One of the elements of any test adaptation is its translation [27, 28]. The NSNS scale has been translated into Polish with the best possible equivalence - both grammatical and semantic. This task was unusually difficult since the NSNS tool contains many items difficult for translation due to their ambiguity, their emotional character or slang use. Remarks made by the Polish patients and the percentage of unanswered items indicate that the Polish respondents had difficulties in understanding the intention of some of the questionnaire items. This proves that further revision of particular items of the questionnaire is required.

Internal consistency of the Polish version of the NSNS was evaluated by analyzing the item-total correlation. The items of the original version reached the values of Spearman's rank correlation coefficient in the range of 0.31-0.69 for "experience" and 0.53-0.82 for "satisfaction". In the Polish version, these values ranged from 0.224 to 0.757 for "experience" and 0.815-0.894 for "sat- 
isfaction". The reliability of the Polish version for "experience" was 0.921, while for "satisfaction" it was 0.981. The original version of the NSNS reached the coefficient value of 0.91 for the "experience" scale and 0.96 for the "satisfaction" scale. Obtained data referring to the psychometric evaluation of the Polish version of the NSNS allowed us to consider both versions as equivalent regarding the psychometric values. The Polish version of the NSNS, similarly to the original, can identify differences referring to "experience" and "satisfaction" with nursing care between particular departments and between hospitals. The source for evaluation of the validity of the Polish version can be the analysis of the connection between the scale results with other data represented by external criteria. In our study, the external criteria consisted of responses to two questions in Part 3 of the questionnaire, which did not belong to the part evaluating "experience" and "satisfaction" with nursing care. The values of Spearman's rank correlation coefficient for the results of the "experience" and "satisfaction" scales were high both for the items referring to general evaluation of the nursing care provided at the department and for the items referring to the total hospitalization period at the department. The results of the correlation analysis confirmed the accuracy of the Polish version of the NSNS.

In conclusion, the Polish version of the NSNS instrument meets the criteria of functional and psychometric equivalence with the original version. It is recommended that some minor changes should be introduced in the wording of the items in the "experience" part of the tool in order to improve the translation quality. It is necessary to continue further studies using the Polish version of the NSNS to ensure continuous verification of reliability and validity of the research tool.

\section{Acknowledgments}

The authors would like to thank the NSNS team at the University of Newcastle for their permission to use the NSNS questionnaire in this study.

\section{References}

1. Jaworowska A, Szutrowa T. General problems of cultural adaptation of tests. In: Cultural adaptation of tests [Polish]. Ciechanowicz A (ed.). Polskie Towarzystwo Psychologiczne - Wydział Psychologii Uniwersytetu Warszawskiego, Laboratorium Technik Diagnostycznych im. Bohdana Zawadzkiego Warszawa 1990.

2. Gaweł G, Twarduś K, Kin-Dąbrowska J, Pyć L. Quality of Nursing`s Care in Cardiologic Department [Polish]. Problemy Pielęgniarstwa 2008; 16: 339-42.

3. Guyatt G. The philosophy of health-related quality of life translation. Qual Life Res 1993; 2: 461-5.

4. Hamrin E. Quality Quality of Life - Methodological Problems Psychometric Measurements in QOL and mea- surements. In: Quality of life in medical science [Polish]. Wołowicka L (ed.). Akademia Medyczna, Poznań 2001.

5. Wild D, Grave A, Marti M, et al. Principles of good practice for the translation and cultural adaptation process for patient-reported outcomes (PRO) measures: Report of the ISPOR Task Force for Translation and Cultural Adaptation. Value in Health 2005; 8: 94-104.

6. Larrabee J, Bolden L. Defining patient-perceived quality of nursing care. J Nurs Care Qual 2001; 16: 34-60.

7. Jelinek R, Haussmann RKD, Hegyvary ST, Newman JE. A methodology for monitoring quality of care. US Department of Health, Education and Welfare. Bethesda. Maryland 1974.

8. Lenartowicz $\mathrm{H}$. The quality management in nursing [Polish]. CEM, Warszawa 1998.

9. Lynn M, Mc Millen B, Sidani S. Understanding and measuring patients' assessment of the quality of nursing care. Nurs Res 2007; 56: 159-66.

10. Moret L, Nguyen J, Pillet N, Falissard B, Lombrail P, Gasquet I. Improvement of psychometric properties of a scale measuring inpatient satisfaction with care: a better response rate and a reduction of the ceiling effect. BMC Health Services Research 2007; 7: 197.

11. Suhonen R, Schmidt L, Radwin L. Measuring individualized nursing care: assessment of reliability and validity of three scales. J Adv Nurs 2007; 59: 77-85.

12. Thomas LH, McColl E, Priest J, Bond S, Boys RJ. Newcastle Satisfaction with Nursing Scale: an instrument for quality assessments of nursing care. Qual Health Care 1996; 5: 67-72.

13. Walsh $M$, Walsh $A$. Measuring patient satisfaction with nursing care: experience of using the Newcastle Satisfaction with Nursing Scale. J Adv Nurs 1999; 29: 307-15.

14. Peterson WE, Charles C, DiCenso A, Sword W. The Newcastle Satisfaction with Nursing Scale: a valid measure of maternal satisfaction with inpatient postpartum nursing care. J Adv Nurs 2005; 52: 672-81.

15. Akin S, Erdogan S. The Turkish version of the Newcastle Satisfaction with Nursing Care Scale used on medical and surgical patients. J Clin Nurs 2007; 16: 646-53.

16. Drwal R. Adaptation of the Personality Questionnaires [Polish]. Brzozowski P (ed.). PWN, Warsaw 1995.

17. Karauda M, Ksykiewicz-Dorota A. Factors influencing on parturients satisfaction with the care provided by midwives in obstetric-neonatal ward [Polish]. Annales Universitatis Mariae Curie-Skłodowska Lublin - Polonia, Sec D. 2005; 60: 359-62.

18. Łukomska A, Rość D, Jachimowicz-Wołoszynek D. The satisfaction of the patient with primary care [Polish]. Annales Academiae Medicae Bydgostiensis 2004; 18: 41-6.

19. Ślusarz R, Jóźwicka M. Evaluation of nursing care in opinion of patients of surgical wards [Polish]. Annales Universitatis Mariae Curie-Skłodowska Lublin-Sec D 2006; 59: 518-22.

20. Burns N, Grove S. The practice of nursing research: conduct, critique and utilization. 2nd ed. W.B. Saunders Company Clearwater 1993.

21. Laschinger H, McGills Hall L, Almost JA. Psychometric analysis of the patient satisfaction with nursing care quality questionnaire. An actionable approach to measuring patient satisfaction. J Nurs Care Qual 2005; 20: 220-30.

22. Mykowska A. Patient Satisfaction and the quality of medical services [Polish]. Zdrowie i Zarządzanie 2002; 4: 69-73.

23. Castle N, Brown J, Hepner K, Hays R. Review of the literature of survey instruments used to collect data on 
hospital patients' perceptions of care. Health Serv Res 2005; 40: 1996-2017.

24. Kulczycka K. The criteria for evaluation of patient satisfaction with nursing care [Polish]. Zdrowie Publiczne 2001; 111: 53-6.

25. Pascoe GC. Patient satisfaction in primary health care. Eval Program Plann 1983; 6: 185-210.

26. Twarduś K, Gaweł G. Perek G. Patients and nurses satisfaction with the quality of nursing care [Polish]. Problemy Pielęgniarstwa 2000; 1: 218-36.

27. Herdman M, Fox-Rushby J, Badia X. 'Equivalence' and the translation and adaptation of health-related quality of life questionnaires. Qual Life Res 1997; 6: 237-47.

28. Hunt SM. Cross-cultural comparability of measures and other issues related to multicountry studies. BJM Econ 1993; 6: 27-34. 